Bogolyubov Institute for Theoretical Physics, Nat. Acad. of Sci. of Ukraine (14b, Metrolohichna Str., Kyiv 03680, Ukraine)

PACS 05.45.-a, 05.45.Pq, 05.65. $+\mathrm{b}$

\title{
SPECTRAL ANALYSIS AND INVARIANT MEASURE IN THE STUDY OF A NONLINEAR DYNAMICS OF THE METABOLIC PROCESS IN CELLS
}

\section{Introduction}

In what follows, we will study the earlier constructed mathematical model of the metabolic process in a cell [1-19]. The model is based on the experimental data concerning bacteria Arthrobacter globiformis at a transformation of steroids [20]. This microorganism is referred to oxygen-breathing bacteria arising 2.48 bln years ago. Due to the evolution of metabolic processes in protobionts, the transition from the oxygenless life of microorganisms to oxygen-breathing bacteria and their subsequent evolution to eukaryotes happened. The relative simplicity of the metabolic process running in given bacteria (the absence of the Krebs cycle, e.g.) allows us to model the metabolic process in a cell on the whole as an open dissipative structure, in which two following basic systems necessary for the life are self-organized: the system of a transformation of the substrate and the respiratory chain. We will consider the specific biochemical process of transformation of steroids by the given type of cells $[2,3]$. This enables us to use the experimentally

(C) V.I. GRYTSAY, 2017 determined parameters in the construction of a model and to make conclusion about structural-functional connections under the self-organization of the given biosystem. If some other substrate is used as a nutrient medium, the mechanism of self-organization of a cell will be analogous.

\section{Mathematical Model}

The mathematical model of the metabolic process in a cell is constructed according to the general scheme of the process of transformation of steroids by given cells (see Fig. 1) and takes the form (1)-(10):

$$
\begin{aligned}
& \frac{d G}{d t}=\frac{G_{0}}{N_{3}+G+\gamma_{2} \psi}-l_{1} V\left(E_{1}\right) V(G)-\alpha_{3} G, \\
& \frac{d P}{d t}=l_{1} V\left(E_{1}\right) V(G)-l_{2} V\left(E_{2}\right) V(N) V(P)-\alpha_{4} P, \\
& \frac{d B}{d t}=l_{2} V\left(E_{2}\right) V(N) V(P)-k_{1} V(\psi) V(B)-\alpha_{5} B, \\
& \frac{d E_{1}}{d t}=E_{10} \frac{G^{2}}{\beta_{1}+G^{2}}\left(1-\frac{P+m N}{N_{1}+P+m N}\right)- \\
& -l_{1} V\left(E_{1}\right) V(G)+l_{4} V\left(e_{1}\right) V(Q)-a_{1} E_{1},
\end{aligned}
$$

ISSN 2071-0194. Ukr. J. Phys. 2017. Vol. 62, No. 5 


$$
\begin{aligned}
& \frac{d e_{1}}{d t}=-l_{4} V\left(e_{1}\right) V(Q)+l_{1} V\left(E_{1}\right) V(G)-\alpha_{1} e_{1} \\
& \frac{d Q}{d t}=6 l V(2-Q) V\left(O_{2}\right) V^{(1)}(\psi)-l_{6} V\left(e_{1}\right) V(Q)- \\
& -l_{7} V(Q) V(N), \\
& \frac{d O_{2}}{d t}=\frac{O_{20}}{N_{5}+O_{2}}-l V(2-Q) V\left(O_{2}\right) V^{(1)}(\psi)-\alpha_{7} O_{2}, \\
& \frac{d E_{2}}{d t}=E_{20} \frac{P^{2}}{\beta_{2}+P 2} \frac{N}{\beta+N}\left(1-\frac{B}{N_{2}+B}\right)- \\
& -l_{10} V\left(E_{2}\right) V(N) V(P)-\alpha_{2} E_{2}, \\
& \frac{d N}{d t}=-l_{2} V\left(E_{2}\right) V(N) V(P)-l_{7} V(Q) V(N)+ \\
& +k_{2} V(B) \frac{\psi}{K_{10}+\psi}+\frac{N_{0}}{N_{4}+N}-\alpha_{6} N \\
& \frac{d \psi}{d t}=l_{5} V\left(E_{1}\right) V(G)+l_{8} V(N) V(Q)-\alpha \psi
\end{aligned}
$$

where $V(X)=X /(1+X), V^{(1)}(\psi)=1 /\left(1+\psi^{2}\right)$, $V(X)$ is a function describing the adsorption of the enzyme in the region of a local coupling, and $V^{(1)}(\psi)$ is a function characterizing the influence of the kinetic membrane potential on the respiratory chain.

In the modeling, it is convenient to use the following dimensionless parameters: $l=l_{1}=k_{1}=0.2$; $l_{2}=l_{10}=0.27 ; l_{5}=0.6 ; l_{4}=l_{6}=0.5 ; l_{7}=1.2$; $l_{8}=2.4 ; k_{2}=1.5 ; E_{10}=3 ; \beta_{1}=2 ; N_{1}=0.03 ;$ $m=2.5 ; \alpha=0.033 ; a_{1}=0.007 ; \alpha_{1}=0.0068$; $E_{20}=1.2 ; \beta=0.01 ; \beta_{2}=1 ; N_{2}=0.03 ; \alpha_{2}=0.02 ;$ $G_{0}=0.019 ; \quad N_{3}=2 ; \gamma_{2}=0.2 ; \alpha_{5}=0.014 ;$ $\alpha_{3}=\alpha_{4}=\alpha_{6}=\alpha_{7}=0.001 ; O_{20}=0.015 ; N_{5}=0.1$; $N_{0}=0.003 ; N_{4}=1 ; K_{10}=0.7$.

Equations (1)-(9) present the changes in the concentrations of: (1) hydrocortisone $(G) ;(2)$ prednisolone $(P) ;(3) 20 \beta$-oxyderivative of prednisolone $(B)$; (4) oxidized form of 3 -ketosteroid- $\Delta^{\prime}$-dehydrogenase $\left(E_{1}\right)$; (5) reduced form of 3 -ketosteroid- $\Delta^{\prime}$-dehydrogenase $\left(e_{1}\right) ;(6)$ oxidized form of the respiratory chain $(Q)$; (7) oxygen $\left(\mathrm{O}_{2}\right)$; (8) 20 $\beta$-oxysteroid-dehydrogenase $\left(E_{2}\right)$; and $(9) \mathrm{NAD} \cdot H$ (reduced form of nicotinamide adenine dinucleotide) $(N)$. Equation (10) describes the change in a level of the kinetic membrane potential $(\psi)$.

The reduction of parameters of the system to the dimensionless form was performed in $[2,3]$.

The presented mathematical model (1)-(10) is studied within the theory of nonlinear differential

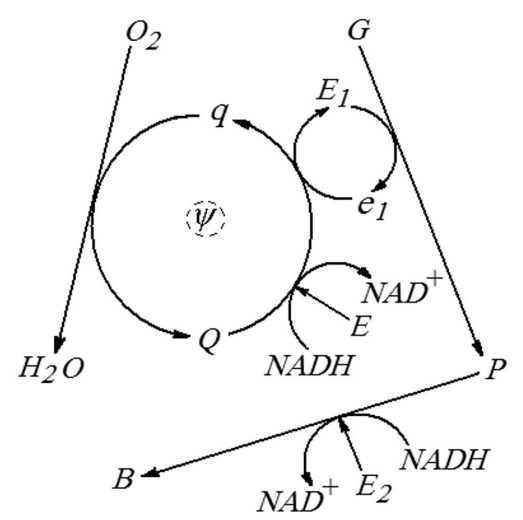

Fig. 1. General scheme of the metabolic process in a cell

equations $[21,22]$ and the methods of simulation of biochemical systems developed by the author and other researchers in [23-52].

This autonomous system of nonlinear differential equations was solved by the Runge-Kutta-Merson method. The exactness of a solution was set to be $10^{-12}$. To ensure the reliability of calculations, namely, the passage of the system from the transient initial phase to the asymptotic solution presented by an attractor, the duration of calculations was taken to be $10^{5}$. In that time, the trajectory "sticks" to the corresponding attractor.

The spectral analysis of the nonlinear dynamics of a metabolic process was carried out by the expansion of the functions describing the kinetics of the system in a trigonometric Fourier series in one of the variables $(G)$. Since we consider the autoperiodic trajectories of regular attractors and strange attractors possessing the fractality, the expansion was performed on the segment $[-l ; l]$. This means that the arbitrarily large period of any autooscillatory mode of the system can be placed on the taken segment $2 l$ in length. The formula of such expansion in a Fourier series reads [53]:

$a_{0}+\sum_{n=1}^{\infty}\left(\left(a_{n} \cos \frac{n \pi x}{l}+b_{n} \sin \frac{n \pi x}{l}\right)\right.$,

where

$$
\begin{aligned}
& a_{0}=\frac{1}{2 l} \int_{-l}^{l} f(x) d(x), \quad a_{n}=\frac{1}{l} \int_{-l}^{l} f(x) \cos \frac{n \pi x}{l} d(x), \\
& b_{n}=\frac{1}{l} \int_{-l}^{l} f(x) \sin \frac{n \pi x}{l} d(x), \quad n \in N .
\end{aligned}
$$




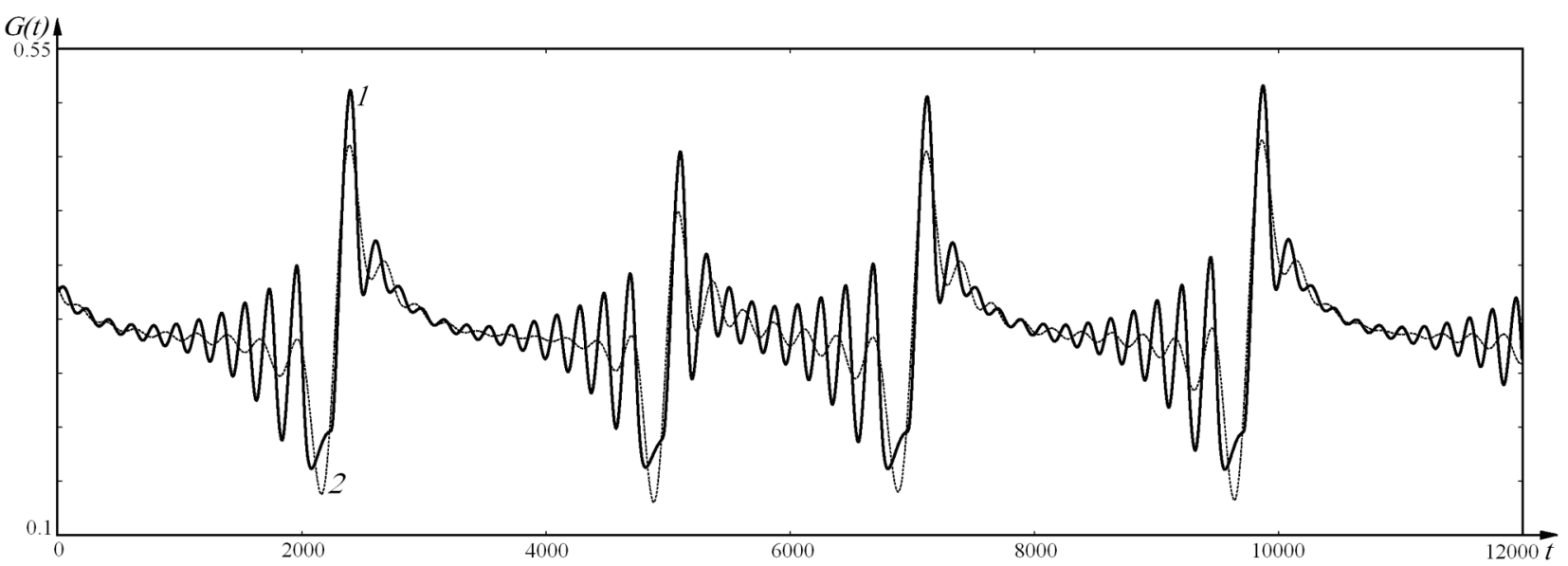

Fig. 2. Summary plot of the kinetics of the variable $G$ in the mode with the strange attractor $13^{*} 2^{x}(\alpha=0.03217)$ formed by the summation of harmonics: $n=1000$ (1) and $n=200$ (2)

The spectrum of Lyapunov indices was calculated with the help of a program written by the author in Fortran. Benettine's algorithm with orthogonalization of vectors by the Gram-Schmidt method was used [21].

As a quantitative measure of the fractality of strange attractors, their Lyapunov dimension was calculated by the Kaplan-Yorke formula $[54,55]$ :

$D_{F_{r}}=m+\frac{\sum_{i=1}^{m} \lambda_{i}}{\left|\lambda_{m+1}\right|}$.

\section{Results of Studies}

While studying the phase-parametric diagram of the system under study, we have got the scenario of adaptation modes of the metabolic process in a cell at a decrease in the dissipation of the kinetic membrane potential $\alpha$ [17]. The calculated plots of the kinetics of modes manifest a stationary behavior and autoperiodic or chaotic oscillations. They reflect the internal dynamics of the metabolic processes in a cell. The possibility of the appearance of autooscillatory modes in the given population of cells was later confirmed experimentally [56].

In order to restrict the volume of the present work, we will consider only separate modes, which does not affect the results. Below, we will consider the scenario of appearance and destroying of autooscillatory modes.

Stationary state $\mapsto 1^{*} 2^{0}(\alpha=0.04131) \mapsto 2^{*} 2^{0}(\alpha=$ $=0.03753) \mapsto 3^{*} 2^{0}(\alpha=0.03563274) \mapsto 5^{*} 2^{0}(\alpha=$ $=0.03463) \mapsto 8^{*} 2^{0}(\alpha=0.033) \mapsto 8^{*} 2^{x}(\alpha=$
$=0.0328709) \mapsto 11^{*} 2^{0}(\alpha=0.032516) \mapsto 11^{*} 2^{x}(\alpha=$ $=0.03239) \mapsto 13^{*} 2^{0}(\alpha=0.03225) \mapsto 13^{*} 2^{x}(\alpha=$ $=0.03217) \mapsto 7^{*} 2^{x} \mapsto 1^{*} 2^{22}(\alpha=0.032161) \mapsto$ $\mapsto 1^{*} 2^{0}(\alpha=0.0321148) \mapsto$ Stationary state.

In order to construct and compare the Fourier spectra on a single scale, the value of $l$ was chosen with regard for the maximally possible reasonable time of representation of the kinetics of the most complicated mode, namely the strange attractor $13^{*} 2^{x}$. In this case, the number of harmonics of the expansion was taken to be $n=1000$. The kinetics of the given mode after the summation of all harmonics is shown in Fig. 2, curve 1. It coincides completely with the initial plot (before the expansion) of the kinetics of the given variable. For $n=200$, the summary plot 2 of harmonics does not coincide with the initial one, which means the smallness of the taken number of harmonics. In what follows, the number of harmonics in expansions in a Fourier series for any mode will be $n=1000$.

In Figs. 3, $a-f$ and $4, a-g$, we show the spectra of expansion in a Fourier series of the variable $G$ for some modes by the above-presented scenario for the variable coefficient $\alpha$. In Fig. $4, h$, we give the spectrum of some mode of a strange attractor for the input parameters $G_{0}=0.009 ; O_{2_{0}}=0.00209$. In the plots, we present the ratio of the amplitudes of harmonics $\hat{G}_{n}$ bounded by a level of 0.03 for the sake of clearness for regular attractors and by a level of 0.014 for strange attractors. Though the calculation of each mode was made for $n=1000$, the plots present only 400 harmonics. All harmonics for $n>400$ are in- 

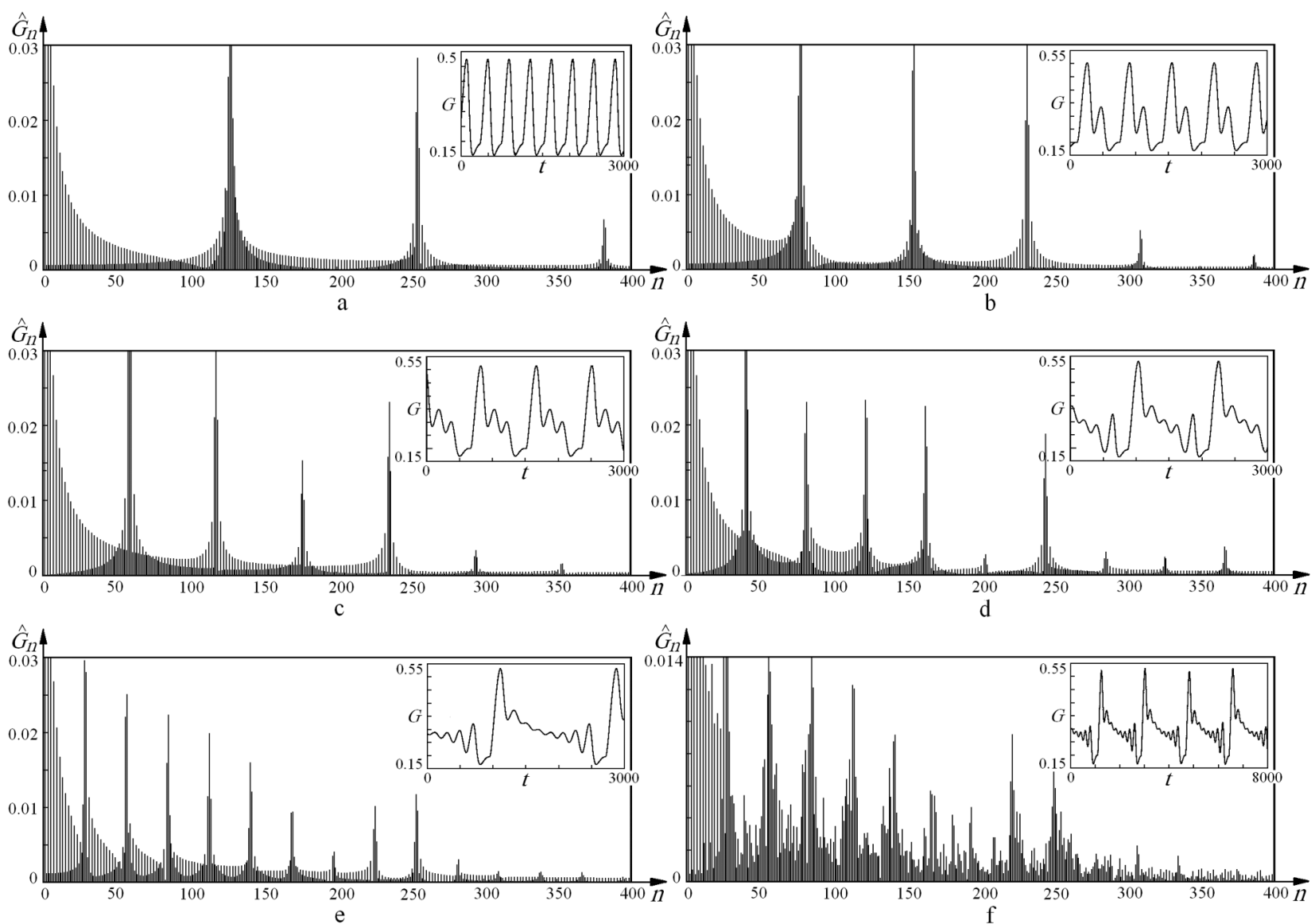

$\hat{G}_{n} \hat{A}$

Fig. 3. Distribution of harmonics of the Fourier spectrum in modes of the metabolic process: regular attractor $1^{*} 2^{0}(\alpha=$ $=0.04131)-a$; regular attractor $2^{*} 2^{0}(\alpha=0.03753)-b$; regular attractor $3^{*} 2^{0}(\alpha=0.03563274)-c ;$ regular attractor $5^{*} 2^{0}(\alpha=$ $=0.03463)-d$; regular attractor $8^{*} 2^{0}(\alpha=0.033)-e$; strange attractor $8^{*} 2^{x}(\alpha=0.0328709)-f$

significant and omitted. In the right upper corner of each plot, we give the kinetic curve of the given variable in this mode.

The plots given correspond to the distribution of spectra of a representation of the corresponding modes. As is seen, a decrease in the dissipation of the kinetic membrane potential in the cyclic metabolic process implies that some harmonics grow and become maximal, whereas other ones decay to a minimum, on the contrary. This complicates the nonlinear dynamics of the process. The decrease in $\alpha$ from 0.04131 to 0.03753 causes a bifurcation and the appearance of the 1-fold (Fig. 3, a) and twofold (Fig. 3, b) periodic modes of a regular attractor. Respectively, this is revealed in the distribution of harmonics. The peaks of the basic harmonics of the expansion vary, and one more peak has arisen. The

ISSN 2071-0194. Ukr. J. Phys. 2017. Vol. 62, No. 5 further decrease in the dissipation of the kinetic membrane potential leads to the appearance of subsequent bifurcations. At $\alpha=0.03563274$, the 3 -fold periodic mode (Fig. 3, c) is formed, the distribution of harmonics is changed, and one more peak appears. As $\alpha$ decreases further, the 4-fold periodic mode arises according to the scenario presented in [17]. Then the 5fold cycle is formed (Fig. 3, d). Further, as a result of bifurcations, the 6-, 7-, and 8-fold periodic cycles appear successively. The spectral distribution for the 8fold cycle is shown in Fig. 3, e. We should like to indicate a change in the spectral pattern at the given time moment. The further decrease in the coefficient of dissipation of the kinetic membrane potential does not lead to the appearance of a bifurcation and the birth of a new cycle, but to the creation of the chaotic mode of the strange attractor (Fig. 3, $f$ ). The Fourier distri- 

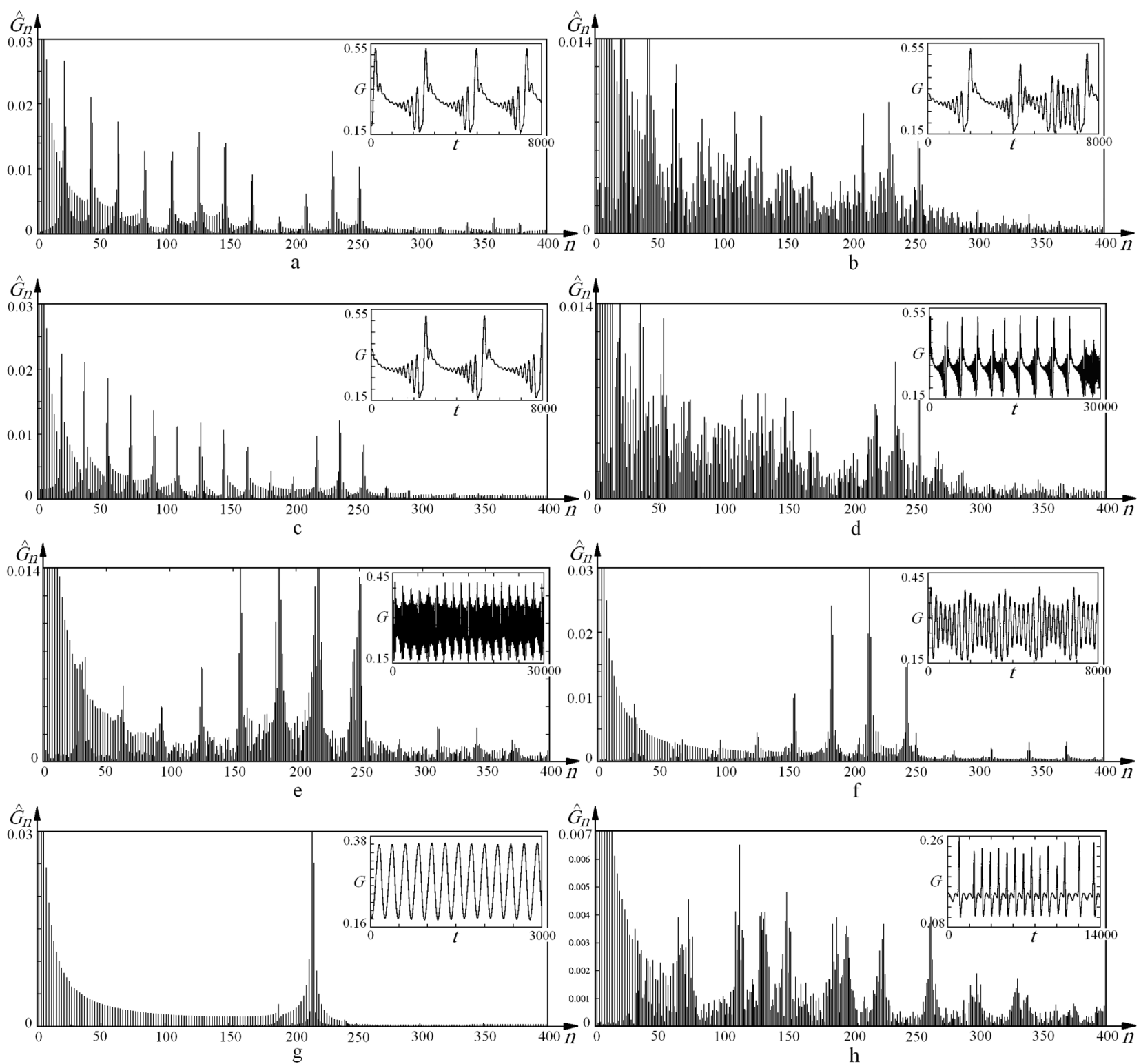

Fig. 4. Distribution of harmonics of the Fourier spectrum in modes of the metabolic process: regular attractor $11^{*} 2^{0}(\alpha=$ $=0.032516)-a$; strange attractor $11^{*} 2^{x}\left(\alpha=0.03239-b\right.$; regular attractor $13^{*} 2^{0}(\alpha=0.03225)-c$; strange attractor $13^{*} 2^{x}(\alpha=$ $=0.03217)-d ;$ strange attractor $7^{*} 2^{x}(\alpha=0.0321646)-e ;$ regular attractor $1^{*} 2^{22}(\alpha=0.032161)-f ;$ regular attractor $1^{*} 2^{0}(\alpha=$ $=0.0321148)-g ;$ strange attractor $3^{*} 2^{x}\left(\alpha=0.033-h ; G_{0}=0.009 ; O_{2_{0}}=0.00209\right)$

bution spectrum becomes more continuous. However, this continuous spectrum includes clearly the harmonics of disappeared limiting cycles. The transition between modes has arisen as a result of the intermittence at the fracture of the laminar part of a trajectory of the 8-fold limiting cycle by the turbulence. The laminar part of the trajectory is formed at the expense of harmonics of the regular attractor $8^{*} 2^{0}$, whereas the turbulence is formed due to the creation of new harmonics. The domain of attraction of the limiting set of the regular attractor is eroded by these harmonics. The further decrease in the coefficient $\alpha$ causes the successive formation of the attractors $9^{*} 2^{0}, 9^{*} 2^{x}, 10^{*} 2^{0}, 10^{*} 2^{x}$, and $11^{*} 2^{0}$. 
The spectral analysis of the last regular attractor is shown in Fig. 4, $a$. It is seen how the selforganization results in a change in the main harmonics of the expansion and in their magnitudes and frequencies. As $\alpha$ decreases to 0.03239 , the selforganization of the given mode is violated, and the chaotic mode of the strange attractor $11^{*} 2^{x}$ is established (see Fig. 4, b). As in the previous case, the positions of the maximal peaks of the basic harmonics of the previous mode $11^{*} 2^{0}$ are conserved. In this case, their magnitudes vary, which testifies to the conservation of the attracting set of the given regular attractor, whereas the appeared additional harmonics violate it slightly. Due to the conservation of maximal harmonics, the trajectory is kept in the domain of attraction of the attractor. The further decrease in $\alpha$ causes the successive formation of the following attractors: $12^{*} 2^{0}, 12^{*} 2^{x}$, and $13^{*} 2^{0}(\alpha=0.03225)$ (Fig. 4, c). The change in the coefficient of dissipation down to 0.03217 causes the destroying of this cycle and the formation of the strange attractor $13^{*} 2^{x}$ (Fig. $4, d$ ). It is the most complicated attractor, for which we searched firstly for the necessary number of harmonics reliably representing the nonlinear dynamics of the process (Fig. 2). As above, the intermittence causes the destroying of the stable 13 -fold periodic cycle. The laminar trajectories of peak harmonics of the attractor $13^{*} 2^{0}$ are mixed with the trajectories of new arisen harmonics creating the turbulence.

The subsequent decrease in $\alpha$ leads, strangely enough, to the appearance of the unstable strange attractor $7^{*} 2^{x}(\alpha=0.0321646)$ (Fig. $\left.4, e\right)$. As a result of the self-organization, the basic bearing frequencies of harmonics vary, and the different type of a 7 -fold limiting cycle, which is the attracting set for chaotic trajectories, is created. The further decrease in $\alpha$ causes the subsequent self-organization and the formation of a 22 -fold autoperiodic cycle $1^{*} 2^{22}(\alpha=$ 0.032161) (Fig. 4, $f$ ). Its expansion spectrum contains a clearly expressed peak of the basic harmonic at a medium frequency. The further decrease in the dissipation of the kinetic membrane potential leads to the establishment of one dominant frequency forming again a 1 -fold periodic mode $1^{*} 2^{0}(\alpha=0.0321148)$ (Fig. $4, g$ ). It passes to a stationary mode, as $\alpha$ decreases. For the comparison of modes, we show the distribution of harmonics of the Fourier spectrum in Fig. $4, h$ for the strange attractor $3^{*} 2^{x}$ formed at $\alpha=0.033 ; G_{0}=0.009 ; O_{2_{0}}=0.00209$. The spectrum of its basic bearing frequencies is essentially different from that in the mode of the regular attractor $3^{*} 2^{0}$ (Fig. 3,c).

The study of the Fourier spectra of modes in Figs. 3, $a-f$ and $4, a-g$ testifies to a geometric similarity of the phase portraits of attractors of the oscillatory dynamics of the system. The redistribution of the amplitudes of harmonics is related to the domains of attraction of that or other attractor. The bearing frequencies characterize the laminar trajectories depending on the multiplicity and the geometric type of an attractor. Their significant change means a change in the domain of attraction of the attractor and, respectively, the geometric type of the attractor (Fig. 4, h).

In Fig. $5, a, b$, we show a projection of the phase portrait of the strange attractor $13^{*} 2^{x}(\alpha=0.03217)$ in the three-dimensional phase space $\left(E_{1}, G, B\right)$ and the histogram of the projection of its invariant measure on the plane $\left(G, E_{1}\right)$.

This strange attractor is formed due to the funnel effect. As is seen from Fig. 5, a, there exists a domain in the phase space, where the mixing of trajectories occurs. An arbitrarily small deviation affects the evolution of the trajectory, and the deterministic chaos is formed. Analogous funnels are formed also for the following strange attractors: $8^{*} 2^{x}, 9^{*} 2^{x}, 10^{*} 2^{x}, 11^{*} 2^{x}$, and $12^{*} 2^{x}$.

In Fig. $6, a-b$, we present the strange attractor $7^{*} 2^{x}(\alpha=0.0321646)$. In this case, as distinct from the previous phase portrait, the funnel of mixing of trajectories is the domain of instability increased to the size of the whole strange attractor. It is a very unstable mode. As $\alpha$ increases insignificantly, the funnel decreases, and the more stable strange attractor $13^{*} 2^{x}$ is established. But then the self-organization results in the appearance of a stabler regular attractor $1^{*} 2^{22}$

As distinct from the above-shown attractors, Fig. 7, $a, b$ presents a strange attractor that is formed not by a change in the coefficient $\alpha$, but by a variation of the input parameters $G_{0}$ and $O_{2_{0}}$. The obtained strange attractor in Fig. 7, $a$ is created as a result of the intermittence of two unstable cycles $3^{*} 2^{x}$ and $2^{*} 2^{x}$ (see the kinetics of $G$ in Fig. $4, h$ ). As a result of the composition of two unstable trajectories, the uncertainty in the evolution of the system arises.

The above-considered phase portraits of the nonlinear dynamics are typical of all studied modes of 


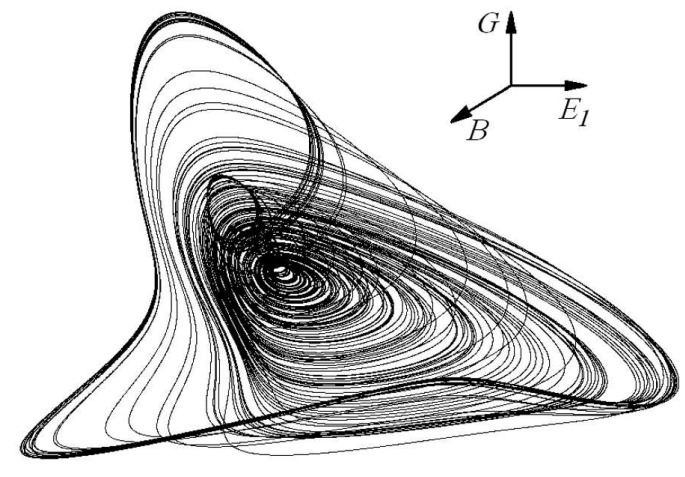

$a$

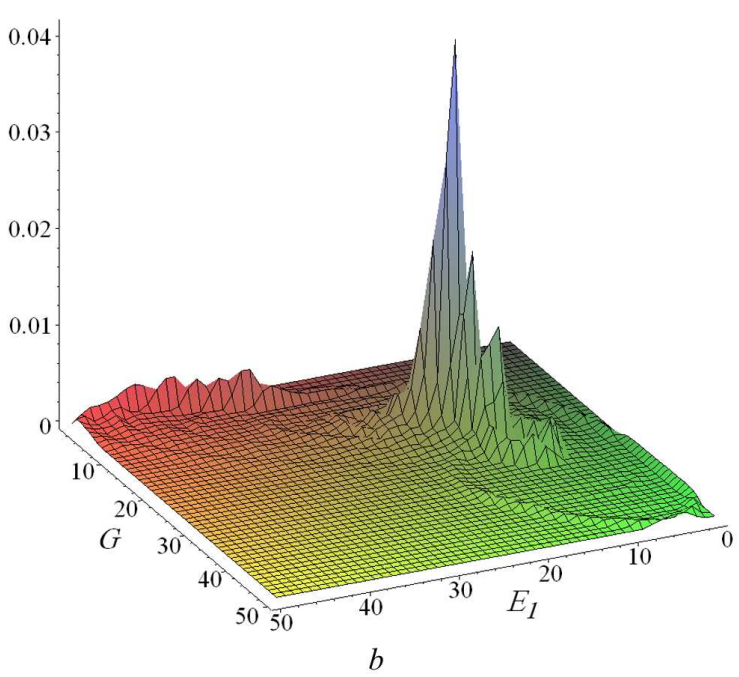

$b$

Fig. 5. Strange attractor $13^{*} 2^{x}(\alpha=0.03217)$ : projection of its phase portrait in the three-dimensional space $\left(E_{1}, G, B\right)-a$; histogram of the projection of the invariant measure on the plane $\left(G, E_{1}\right)-b, t \in\left(10^{6}-10^{6}+5 \times 10^{5}\right)$

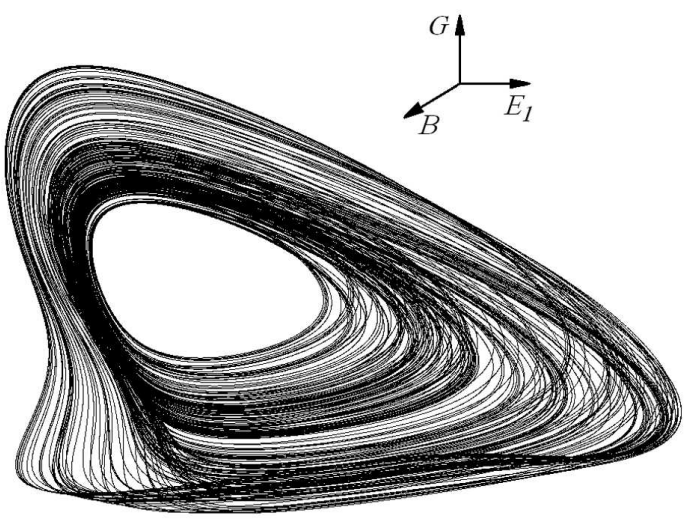

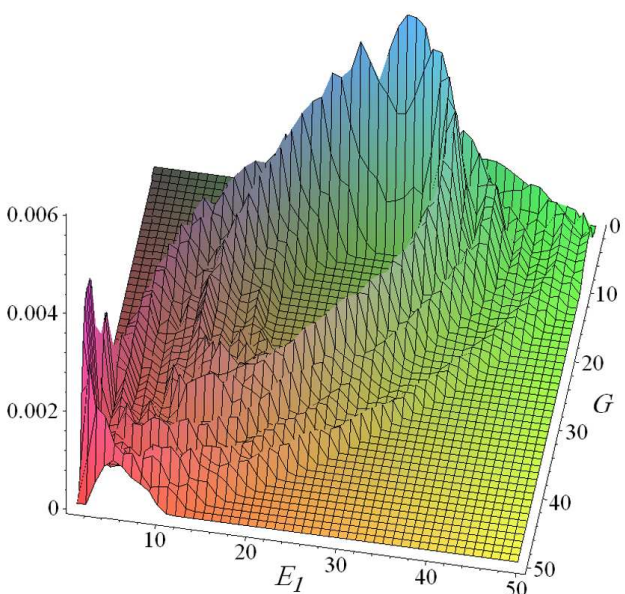

$b$

Fig. 6. Strange attractor $7^{*} 2^{x}(\alpha=0.0321646)$ : projection of its phase portrait in the three-dimensional space $\left(E_{1}, G, B\right)-a$; histogram of the projection of the invariant measure on the plane $\left(G, E_{1}\right)-b, t \in\left(10^{6}-10^{6}+5 \times 10^{5}\right)$

strange attractors of our system. In Figs. $5, b, 6, b$, and $7, b$, we constructed the histograms of projections of their invariant measures. To make it, the phase spaces of the given strange attractors were partitioned into the number of boxes, which is maximally possible for the computer program, and the probabilities of the attendance of each box by the trajectory were calculated. The numbers of points of the mapping for the whole phase portrait were taken to be 500,000 in Figs. 5, $b$ and $6, b$ and 2,000,000 in Fig. $7, b$. The numerical experiment showed that a change in the numbers of points of the mapping has no influence on the probability of the attendance of each box by the trajectory. The time shift along a trajectory $(x(t) \rightarrow x(t+\tau))$ has no influence as well, which means the invariance of the measures of the given strange attractors.

The peak of the invariant measure in Fig. $5, b$ characterizes visually the attracting set of the given attractor in the funnel, and the ability to mix its trajectories in this compressed domain of the phase space. Figure $6, b$ contains no such domain. The mixing occurs over the whole domain of the phase space.

ISSN 2071-0194. Ukr. J. Phys. 2017. Vol. 62, No. 5 


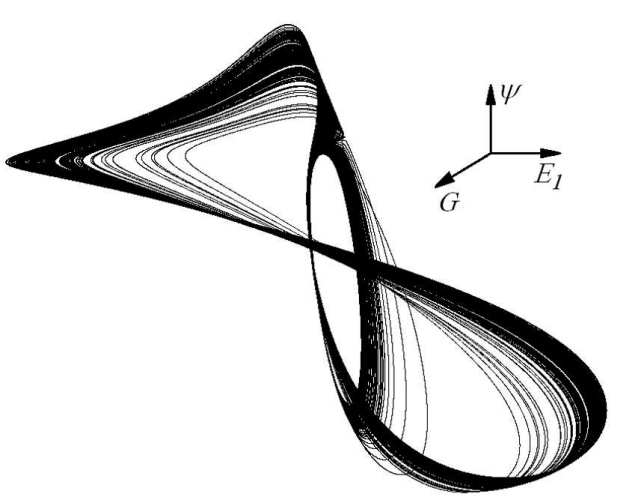

$a$

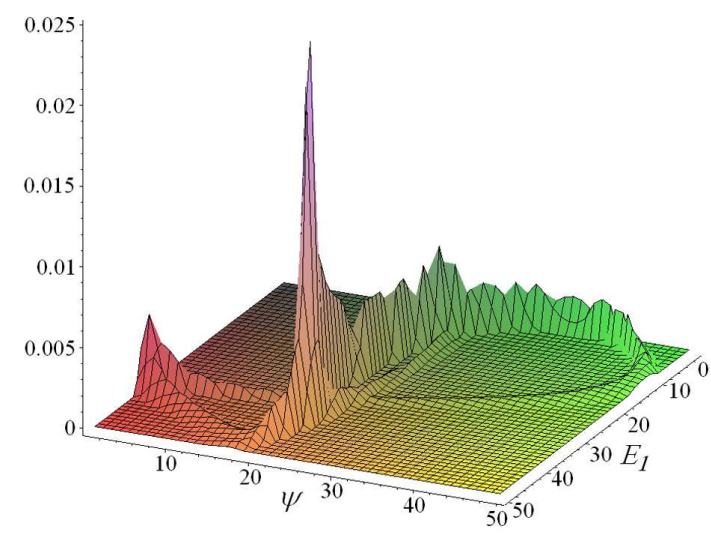

$b$

Fig. 7. Strange attractor $3^{*} 2^{x}\left(\alpha=0.033 ; G_{0}=0.009 ; O_{2_{0}}=0.00209\right)$ : projection of its phase portrait in the three-dimensional space $\left(E_{1}, \psi, G\right)-a$; histogram of the projection of the invariant measure on the plane $\left(E_{1}, \psi\right)-$ $b, t \in\left(10^{6}-10^{6}+2 \times 10^{6}\right)$

\section{Lyapunov's exponents of strange attractors of the system}

\begin{tabular}{|l|c|c|c|c|c|c|c|}
\hline \multicolumn{1}{|c|}{$\alpha$} & Attractor & $\lambda_{1}$ & $\lambda_{2}$ & $\lambda_{3}$ & $\lambda_{10}$ & $\Lambda$ & $D_{F_{r}}$ \\
\hline 0.032872 & $8^{*} 2^{x}$ & 0.00013 & 0.00000 & -0.00506 & -0.52172 & -0.93352 & -2.02569 \\
0.03268 & $9^{*} 2^{x}$ & 0.00049 & 0.00000 & -0.00500 & -0.51658 & -0.92495 & -2.0980 \\
0.03254 & $10^{*} 2^{x}$ & 0.00026 & 0.00000 & -0.00459 & -0.50965 & -0.91713 & -2.05664 \\
0.03242 & $11^{*} 2^{x}$ & 0.00035 & 0.00000 & -0.00449 & -0.50683 & -0.91260 & -2.07795 \\
0.032278 & $12^{*} 2^{x}$ & 0.00060 & 0.00000 & -0.00402 & -0.50460 & -0.90649 & -2.14925 \\
0.03217 & $13^{*} 2^{x}$ & 0.00083 & 0.00000 & -0.00361 & -0.50326 & -0.90210 & -2.23075 \\
0.0321646 & $7^{*} 2^{x}$ & 0.00031 & 0.00000 & -0.00111 & -0.51650 & -0.90393 & -2.27928 \\
\hline
\end{tabular}

For the above-considered scenario of formation and destroying of strange attractors, we calculated the complete spectra of Lyapunov's exponents and the Lyapunov dimensions of their fractality (Table) (indices $\lambda_{4}-\lambda_{9}$ are omitted, since they do not influence the meaning of the presented results).

According to the data presented in Table, the KS-entropy (Kolmogorov-Sinai entropy) [57] of the strange attractor $13^{*} 2^{x}$ is maximal and equals 0.00083 . For the attractor $7^{*} 2^{x}$, it is equal to 0.00031 . This means that the exponential divergence of phase trajectories along the vector of perturbations corresponding to $\lambda_{1}$ for the strange attractor $13^{*} 2^{x}$ is larger than for the attractor $7^{*} 2^{x}$. For the rest vectors corresponding to negative values of $\lambda_{3}-\lambda_{10}$, the phase trajectories approach exponentially their attractor. Respectively, the nonpredictability and chaoticity of the deterministic chaos for the first strange attractor are higher than for the second one. The larger the value of KS-entropy, the more complicated is the structure of a chaos. At the same time, the ratio of the Lyapunov dimensions of the fractality of these modes is opposite. We have $D_{F_{r}}=-2.23075$ for the strange attractor $13^{*} 2^{x}$ and $D_{F_{r}}=-2.27928$ for $7^{*} 2^{x}$. This can be explained by that the Lyapunov dimensions for these modes are defined not only by the values of $\lambda_{1}$ and $\lambda_{2}$, but also by $\left|\lambda_{3}\right|$. This characterizes a deformation of an element of the phase volume along the corresponding vectors of perturbations. The larger $\lambda_{1}$ and the less $\left|\lambda_{3}\right|$, the larger is the deformation of its volume.

The studies showed that the domain of instability of autooscillatory modes is located between two stationary modes of the metabolic process. It appears due to the breaking of a synchronization between the system of consumption of a substrate and the respiratory chain. This breaking occurs due to the expenditure of the proton membrane potential of a cell for other metabolic processes. A decrease in the potential causes the desynchronization of the processes of 
consumption of a substrate and the processes of transport and accumulation of a charge on the outer side of the membrane. The blocking of the respiratory chain by an increased level of the potential is decreased, the desynchronization arises, and autoperiodic or chaotic oscillations in the metabolic process appear.

The executed expansions in a Fourier series of the determined autooscillatory modes allow us to substantiate this method for the seeking and the identification of autoperiodic and chaotic modes in the metabolic process in a cell. The calculated histograms of the invariant measures of strange attractors give a more complete visual representation of the domains, where the trajectories are mixed, as compared with the phase portraits.

\section{Conclusions}

In the frame of the mathematical model, the autooscillatory modes of the metabolic process in a cell are studied with the help of the expansion of the kinetics of the process in a Fourier series and the construction of histograms of the invariant measures of chaotic attractors of the model. We determined the necessary number of harmonics, which represent uniquely the most complicated mode of a strange attractor. The dependence of the type of an attractor on the distributions and the amplitudes of harmonics in the Fourier spectrum is investigated. The harmonics forming the laminar and turbulent parts of a trajectory of the attractor are identified. The histograms of projections of the invariant measures of the main types of strange attractors of the system are constructed, and their dependence on the phase portrait is determined. The complete spectra of Lyapunov's exponents, KS-entropy, and Lyapunov dimensions of the fractality for the strange attractors under study are calculated. The mechanisms and the causes for the appearance of autoperiodic and chaotic oscillations in the metabolic process in a cell are found.

The work is supported by the project No.0113U001093 of the National Academy of Sciences of Ukraine.

1. V.P. Gachok, V.I. Grytsay. Kinetic model of macroporous granule with the regulation of biochemical processes. Dokl. Akad. Nauk SSSR 282, No. 1, 51 (1985).

2. V.P. Gachok, V.I. Grytsay, A.Yu. Arinbasarova, A.G. Medentsev, K.A. Koshcheyenko, V.K. Akimenko. Kinetic model of hydrocortisone 1-en-dehydrogenation by Arth- robacter globiformis. Biotechn. Bioengin. 33, 661 (1989) [DOI: 10.1002/bit.260330602].

3. V.P. Gachok, V.I. Grytsay, A.Yu. Arinbasarova, A.G. Medentsev, K.A. Koshcheyenko, V.K. Akimenko. Kinetic model for the regulation of redox reaction in steroid transformation by Arthrobacter globiformis cells. Biotechn. Bioengin. 33, 668 (1989) [DOI: 10.1002/bit.260330603].

4. V.I. Grytsay. Self-organization in the macroporous structure of the gel with immobilized cells. Kinetic model of the bioselective membrane of a biosensor. Dopov. Nats. Akad. Nauk Ukr. No. 2, 175 (2000).

5. V.I. Grytsay. Self-organization in a reaction-diffusion porous media. Dopov. Nats. Akad. Nauk Ukr. No. 3, 201 (2000).

6. V.I. Grytsay. Ordered structure in a mathematical model of biosensor. Dopov. Nats. Akad. Nauk Ukr. No. 11, 112 (2000).

7. V.I. Grytsay. Self-organization of the biochemical process in immobilized cells of the bioselective membrane of a biosensor. Ukr. J. Phys. 46, 124 (2001).

8. V.V. Andreev, V.I. Grytsay. Modeling of inactive zones in porous granules of a catalyst and in a biosensor. Matem. Modelir. 17, No. 2, 57 (2005).

9. V.V. Andreev, V.I. Grytsay. Influence of heterogeneity of the diffusion-reaction process for the formation of structures in the porous medium. Matem. Modelir. 17, No. 6, 3 (2005).

10. V.I. Grytsay, V.V. Andreev. The role of diffusion in the active structures formation in porous reaction-diffusion media. Matem. Modelir. 18, No. 12, 88 (2006).

11. V.I. Grytsay. Unsteady conditions in porous reactiondiffusion. Roman. J. Biophys. 17, No. 1, 55 (2007).

12. V.I. Grytsay. The uncertainty in the evolution structure of a reaction-diffusion medium bioreactor. Biofiz. Visn. 19, No. 2, 92 (2007).

13. V.I. Grytsay. Morphogenetic field forming and stability of bioreactor immobilization cells. Biofiz. Visn. 20, No. 1, 48 (2008).

14. V.I. Grytsay. Prediction structural instability and type of the attractor of a biochemical process. Biofiz. Visn. 23, No. 2, 77 (2009).

15. V.I. Grytsay. Structural instability of a biochemical process. Ukr. J. Phys. 55, No. 2, 599 (2010).

16. V.I. Grytsay, I.V. Musatenko. Self-oscillatory dynamics of the metabolic process in a cell. Ukr. Biochem. J. 85, No. 2, 93 (2013) [DOI: 10.15407/ubj85.02.093].

17. V.I. Grytsay, I.V. Musatenko. The structure of a chaos of strange attractors within a mathematical model of the metabolism of a cell. Ukr. J. Phys. 58, No. 7, 677 (2013) [DOI: 10.15407/ujpe58.07.0677].

18. V. Grytsay, I. Musatenko. A mathematical model of the metabolism of a cell. Self-organization and chaos. Chaotic Modeling and Simulation (CMSIM) No. 4, 539 (2013). 
19. V.I. Grytsay, I.V. Musatenko. Self-organization and chaos in the metabolism of a cell. Biopolym. Cell 30 No. 5, 403 (2014) [DOI: 10.7124/bc.0008B9].

20. A.A. Akhrem, Yu.A. Titov. Steroids and Microorganisms (Nauka, 1970) (in Russian).

21. S.P. Kuznetsov. Dynamical Chaos (Fizmatlit, 2001) (in Russian).

22. V.S. Anishchenko. Complex Oscillations in Simple Systems (Nauka, 1990) (in Russian).

23. Yu.M. Romanovskii, N.V. Stepanova, D.S. Chernavskii. Mathematical Biophysics (Nauka, 1984) (in Rissian).

24. G.G. Malinetskii, A.B. Potapov. Modern Problems of Nonlinear Dynamics (Editorial URSS, 2002) (in Russian).

25. E.E. Sel'kov. Self-Oscillations in Glycolysis. Europ. J. Biochem. 4, 79 (1968) [DOI: 10.1111/j.14321033.1968.tb00175.x].

26. M. Holodniok, A. Klic, M. Kubicek, M. Marek. Methods of Analysis of Nonlinear Dynamical Models (Academia, 1986) (in Czech).

27. G.Yu. Riznichenko. Mathematical Models in Biophysics and Ecology (Inst. of Computer. Studies, 2003) (in Russian).

28. V.S. Podgorskij. Physiology and Metabolism of MethanolAssimilating Yeast (Naukova Dumka, 1982) (in Russian).

29. V. Anishchenko, V. Astakhov, A. Neiman, T. Vadicasova, L. Schimansky-Geir. Nonlinear Dynamics of Chaotic and Stochastic System. Tutorial and Modern Developments (Springer, 2007) [ISBN: 978-3-540-38168-6].

30. Chaos in Chemical and Biochemical Systems. Ed. by R. Field, L. Gyorgyi (World Scientific, 1993).

31. V.A. Kordium, D.M. Irodov, O.O. Maslova, T.A. Ruban, E.M. Sukhorada, V.I. Andrienko, N.S. Shuvalova, L.I. Likhachova, S.P. Shpilova. Fundamental biology reached a plateau - development of ideas. Biopolymers $\&$ Cells $\mathbf{2 7}$ (6), 480 (2011).

32. V.I. Grytsay. The conditions of the self-organization in the multienzyme prostacyclin-thromboxane system. Visn. Kyiv. Univ. No. 3, 372 (2002).

33. V.I. Grytsay, V.P. Gachok. The modes of self-organization B prostacyclin-thromboxane system. Visn. Kyiv. Univ. No. 4, 365 (2002).

34. V.I. Grytsay, V.P. Gachok. Ordered structures in the mathematical system of prostacyclin and thromboxane model. Visn. Kyiv. Univ., Ser. Fiz.-Mat. Nauk. No. 1, 338 (2003).

35. V.I. Grytsay. Modeling of processes in the multienzyme prostacyclin and thromboxane system. Visn. Kyiv. Univ. No. 4, 379 (2003).

36. V.P. Gachok, Kinetics of Biochemical Processes. (Naukova Dumka, Kiev, 1988) (in Russian).

37. V.P. Gachok. Strange Attractors in Biosystems (Naukova Dumka, 1989) (in Russian).

38. S.D. Varfolomeev, A.T. Mevkh, V.P. Gachok. Kinetic model of the multienzyme system of blood prostanoid synthesis. 1. Mechanism of stabilization of the levels of thromboxane and prostacyclin. Molek. Biol. 20, No. 4, 957 (1986).

39. S.D. Varfolomeev, V.P. Gachok, A.T. Mevkh. Kinetic behavior of the multienzyme system of blood prostanoid synthesis. BioSystems 19, 45 (1986).

40. V.I. Grytsay, I.V. Musatenko. Self-organization and fractality in metabolic processes of the Krebs cycle. Ukr. Biokhim. Zh. 85, No. 5, 191 (2013).

41. V. Grytsay, I. Musatenko. Nonlinear self-organization dynamics of a metabolic process of the Krebs cycle. Chaotic Modeling and Simulation (CMSIM) 3, 207 (2014).

42. V. Grytsay. Lyapunov indices and the Poincare mapping in a study of the stability of the Krebs cycle. Ukr. J. Phys. 60, No. 6, 561 (2015) [DOI: 10.15407/ujpe60.06.0561].

43. V.I. Grytsay. Self-organization and fractality in the metabolic process of glycolysis. Ukr. J. Phys. 60, No. 12, 1243 (2015) [DOI: 10.15407/ujpe60.12.1251].

44. V.I.Grytsay. Self-organization and chaos in the metabolism of hemostasis in a blood vessel. Ukr. J. Phys. 61, No. 7, 648 (2016) [DOI: 10.15407/ujpe61.07.0648].

45. V.I. Grytsay. A mathematical model of the metabolic process of atherosclerosis. Ukr. Biochem. J. 88, No. 4, 75 (2016) [DOI: 10.15407/ubj88.04.075].

46. V. Grytsay. Self-organization and fractality created by gluconeogenesis in the metabolic process. Chaotic Modeling and Simulation (CMSIM) 2, 113 (2016).

47. A. Golub, O. Matyshevska, S. Prylutska, V. Sysoyev, L. Ped, V. Kudrenko, E. Radchenko, Yu. Prylutskyy, P. Scharff, T. Braun. Fullerenes immobilized at silica surface: topology, structure and bioactivity. J. Mol. Liq. 105, No. 2-3, 141 (2003) [DOI: 10.1016/S01677322(03)00044-8].

48. Yu.I. Prylutskyy, V.M. Yashchuk, K.M. Kushnir, A.A. Golub, V.A. Kudrenko, S.V. Prylutska, I.I. Grynyuk, E.V. Buzaneva, P. Scharff, T. Braun, O.P. Matyshevska. Biophysical studies of fullerene-based composite for bionanotechnology. Mater. Sci. Engineer. C 23, Nos. 1-2, 109 (2003) [DOI: 10.1016/S0928-4931(02)00244-8].

49. A.D. Suprun, Yu.I. Prylutskyy, A.M. Shut, M.S. Miroshnichenko. Towards a dynamical model of skeletal muscle. Ukr. J. Phys. 48, No. 7, 704 (2003).

50. Yu.I. Prylutskyy, A.M. Shut, M.S. Miroshnychenko, A.D. Suprun. Thermodynamic and mechanical properties of skeletal muscle contraction. Int. J. Thermophys. 26, No. 3, 827 (2005) [DOI: 10.1007/s10765-005-5580-8].

51. A.D. Suprun, A.M. Shut, Yu.I. Prylutskyy. Simulation of the Hill equation for fiber skeletal muscle contraction. $U k r$. J. Phys. 52, No. 10, 997 (2007).

52. M. Zabolotnyy, Yu. Barabash, Yu. Sklyarov, Yu. Prylutskyy. The model of photoinduced changes in the pigmentprotein complex of reaction center. Ukr. Bioorg. Acta No. 1, 27 (2010). 
53. N.S. Piskunov. Differential and Integral Calculi (Nauka, 1978) (in Russian).

54. J.L. Kaplan, J.A. Yorke. The onset of chaos in a fluid flow model of Lorenz. Ann. N. Y. Acad. Sci. 316, 400 (1979) [DOI: 10.1111/j.1749-6632.1979.tb29484.x].

55. J.L. Kaplan, J.A. Yorke. Chaotic behavior of multidimensional difference equations. In Functional Differential Equations and Approximations of Fixed Points, edited by H.O. Peitgen, H.O. Walther (Springer, 1979), p. 204.

56. A.G. Dorofeev, M.V. Glagolev, T.F. Bondarenko, N.S. Panikov. Unusual growth kinetics of Arthrobacter globiformis and its explanation. Mikrobiol. 61, 33 (1992).

57. Ya.B. Pesin. Characteristic Lyapunov indices and the ergodic theory. Usp. Mat. Nauk 32, No. 4, 55 (1977).

Received 08.02.17
В.Й. Грицай

СПЕКТРАЛЬНИЙ АНАЛІЗ ТА ІНВАРІАНТНА МІРА ПРИ ДОСЛІДЖЕННІ НЕЛІНІЙНОЇ ДИНАМІКИ МЕТАБОЛІЧНОГО ПРОЦЕСУ В КЛІТИНІ

$\mathrm{P}$ е $з$ ю м е

Проведено моделювання метаболічного процесу в клітині 3 використанням перетворення Фур'є та побудови гістограм інваріантних мір хаотичних атракторів. Зокрема, знайдено сценарій адаптації метаболічного процесу при зміні дисипації кінетичного мембранного потенціалу, послідовність режимів самоорганізації та детермінованого хаосу і, відповідно, розглянуто спектральне відображення з атракторами цих режимів. Проаналізовано структурно-функціональні зв'язки метаболічного процесу в клітині як єдиної дисипативної системи. 